\title{
DISTRIBUTED MICROCONTROLLER BASED CONTROL SCHEME FOR REMOTE CONTROL OF FEL POWER SUPPLY
}

\author{
Neha Modi ${ }^{1}$ \\ ${ }^{1}$ Mahakal Institute of Technology/Electronics and Communication, Ujjain, India
}

\begin{abstract}
Infrared Free Electron Laser (IRFEL) which is under development at RRCAT Indore. The IRFEL machine consists of 90keV thermionic gun as electron source, beam transport line, 25MeV Linear Accelerator (LINAC) and an undulator magnet. There are various magnets on beam transport line. These magnets are energized by power supplies. These power supplies have local as well as remote control and located at equipment hall. The control room and equipment hall are at approximate distance of $300 \mathrm{~m}$. When in operation Free Electron Laser generates X-ray radiations which are harmful for human body. Hence, when FEL is operational no personnel can enter FEL hall. Therefore, power supply kept in this hall needs a remote operation. In this paper, a distributed control system hardware and parameter display for Controlling and monitoring of power supply is designed based on network management protocol i.e. Modbus protocol makes the system flexible for Humans
\end{abstract}

Keywords: Remote monitoring, hardware scheme, Labview, Modbus Protocol

\section{INTRODUCTION}

To operate the magnets involved in the IRFEL system [1] it needs some power supplies, these power supplies are located nearby to the system, as the system radiates harmful rays no personnel is allowed to enter to the equipment hall where the system is operating so it is very necessary to control the power supplies from the remote location via a computer (master PC). The control system has to provide a stable set-point to the power supplies. The read back signal of each of the power supply has to be monitored continuously. Each power supply has three digital inputs and one analog input. Three digital inputs can be used to control power supply ON, OFF and Fault reset whereas analog input sets the power supply current. There are three status signals provided by power supply. They are Power supply ON/OFF, Fault indication, Remote/Local indication.

This Distributed network system consists of one personal computer "Master computer" located in control room for remote operation of the power supplies and two numbers of the "slave control module". Master computer has a Front end GUI which enables the operator to control and monitor power supply parameters and thus facilitates Human machine Interface, HMI [2]

Each slave control module has a MCS-51 family microcontroller, analog and digital I/O and RS-485 serial interface. Each slave controller controls four power supplies. Each slave controller has a unique address called the SLAVE ID. A Master computer provides Interface for machine operation from a remote control room. These slave controllers were connected to Master computer in a bus topology via RS-485 bus. Modbus protocol for the software development in distributed network is described in detail.

\section{REMOTE CONTROL SCHEME}

The LASER system involves magnets which requires power supplies for its operation, these power supplies is operating on very high voltage i.e. about $3.5 \mathrm{kV}$ peak, to control such high voltage power supplies remotely with a PC which is actually operating on low voltage requires some circuits that can establish the communication between the power supplies and the PC and also isolates the high voltage circuit i.e. Magnet power supply with low voltage circuit i.e. Master PC. The circuits which do this interfacing work is nothing but the microcontroller card, Digital Input Output card, ADC card, DAC card, each card with proper optical isolation with it. Using these circuits the development of a Distributed system is designed, which connects largely distributed controllers to centralized PC located at control room which is away from distributed devices. Hence the Micro-controller 8051 based controllers is used which are on a RS-485 network. These network controllers are controlled from a Master PC [3].

These distributed devices are being controlled has an individual control Module called as "Slave controller" which includes a MCS -51 family microcontroller board, Analog to digital convertor (ADC board), Digital to analog convertor (DAC board), and Digital I/Os board. These slave devices are connected to Master PC called as "Master controller". Connection between slave devices and master is on two wire RS-485 bus. Master controller provides a Human Machine Interface (HMI). So all Digital outputs, analog outputs can be set from master controller, similarly ana$\log \&$ digital data from the field will be read by slave controller and sent to master controller. Hence slave controller operations can be controlled from a remote control location. For automation, Master/slave architecture is implemented in which MODBUS protocol [4] is used for data communication between slave controller and a master controller. 


\section{HARDWARE SCHEME}

The hardware scheme of the slave controller module is described in this section: The slave controller design is based on MCS-51 family of micro-controllers. This architecture is chosen since it is an industry standard 8-bit controller with several manufacturing sources and has wide variety of onchip peripherals ideally suited for control applications. This architecture is versatile and has many on chip peripherals. The power supply to be controlled has an analog set-point input, an analog read-back output signal and few digital I/O signals for on/off control and indicators as their (remote) control interface.

In order to meet the generalized requirement of each subsystem and to have flexibility and configurability, the design of the slave controller hardware module was partitioned into various plug-in boards on backplane bus viz. micro-controller board, digital input/output board, analog input/output board, signal conditioning board, etc.

\subsection{Micro-Controller Board}

The micro-controller board is based on Phillips make P89C51RD26 [9]. This board has a LCD interface, and a RS-232 serial interface. A block schematic for the board is shown in figure 1. This board can be plugged onto a 64-pin Euro backplane bus with proprietary design; to provide interface with additional peripheral boards. The microcontroller has on chip 64KB flash program memory. This memory can be programmed through parallel or serial interface.

The serial programming can be done in two modes namely in-system programming (ISP) or in-application programming (IAP). ISP mode allows the user to download new firmware code without chip removal, while in IAP user can upgrade the firmware at run time. This device executes one machine cycle in 6 clock states; hence provides twice the execution speed compared to a conventional MCS-51 devices. A one-time programmable (OTP) configuration bit lets the user select conventional 12 clock state execution, if desired. The instruction set is $100 \%$ binary compatible with the MCS-51 instruction set. The device has four 8-bit input/output ports, three 16-bit timer/event counters, a watchdog timer, an enhanced UART and on-chip oscillator and timing circuits.

The CPU board has $32 \mathrm{kB}$ scratch pad RAM memory. The remaining $32 \mathrm{kB}$ address space is used for interfacing $\mathrm{I} / \mathrm{O}$ devices like ADC, DAC and digital I/O. This board provides buffered data and address bus for interfacing peripheral devices to micro-controller. On the backplane bus there is interfacing signals like power signal, data/address bus, board selection control line, buffered clock etc.

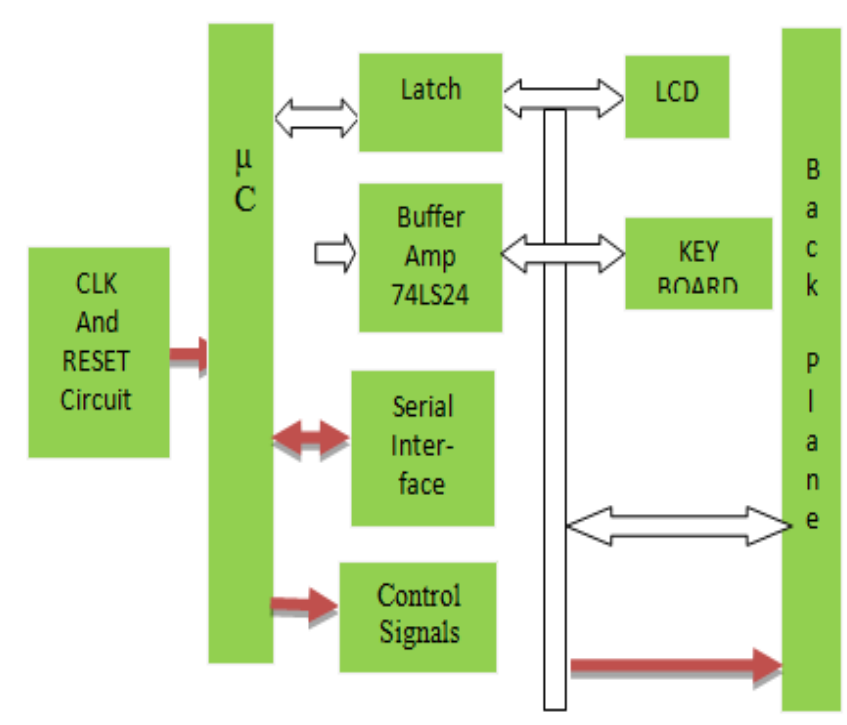

Fig 1: Microcontroller Board

\subsection{Digital Input / Output Board}

The digital input/output (DIO) board is based on Programmable Peripheral Interface (PPI) chip 8255[9]. The board layout is shown in Fig-2. Each PPI has three 8- bit TTLcompatible ports, which can be configured as either input or output. This board provides 24-bit individual outputs/inputs, or any mixture of inputs or outputs. The 8255 chip provides three distinct operating modes (Modes 0, 1 and 2). In this application, mode 0 is used for all the ports. Using mode 0; we have configured Port A \& Port C (upper half) as output ports; while Port B \& Port C (lower half) as input ports. Thus there are 12 inputs and 12 outputs per digital I/O board.

Since input and output ports are interfaced with high voltage and high current systems, they are isolated to avoid ground bounce, using Opto-coupler MCT2E. For easy diagnostics, LED indication is provided on both; input as well as output channels. These LED indicators are located on the front panel of the slave controller. 


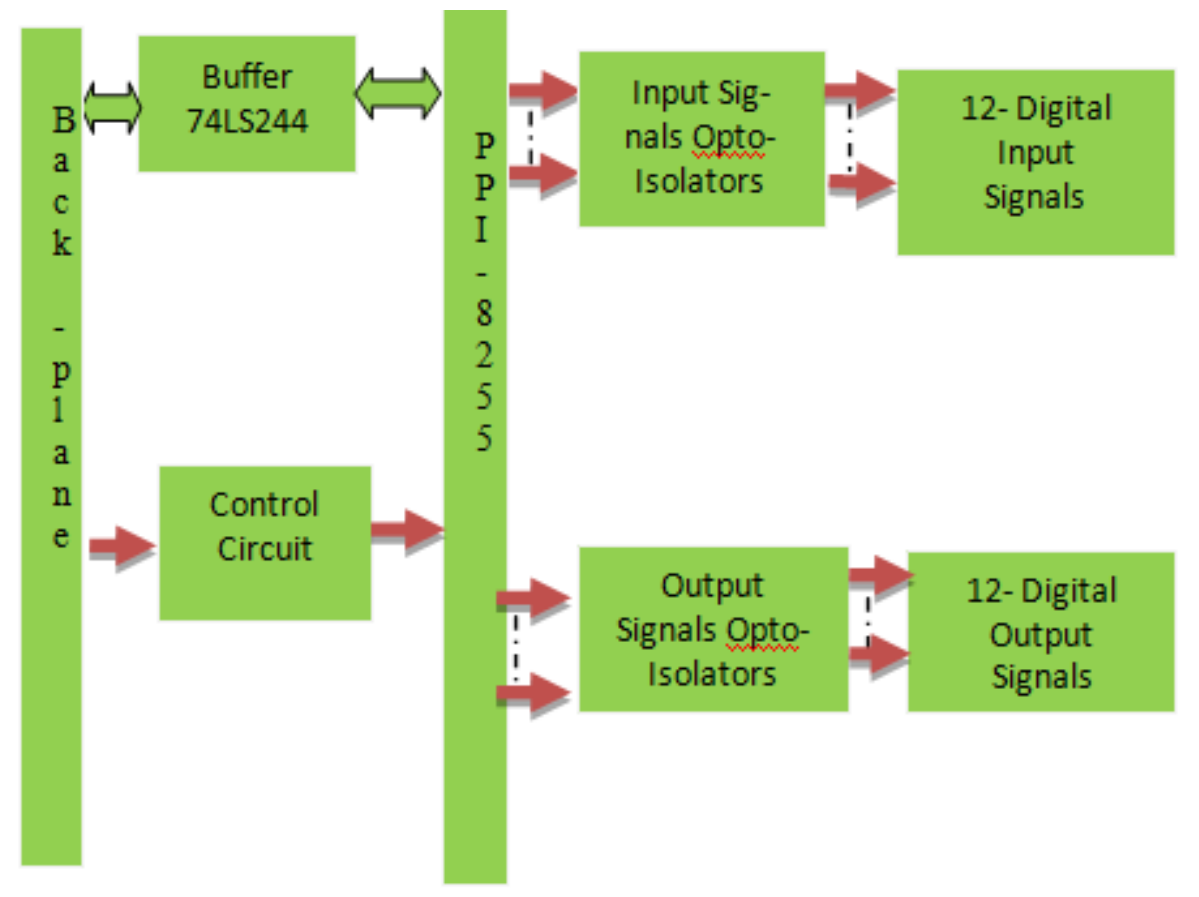

Fig 2: Digital Input / Output Board

Digital I/O signals provide the necessary control signals for on/off individual power supplies, and fault reset control. Few digital signals are used for monitoring their status information like power supply ON/OFF, Local/Remote and fault.

\subsection{Digital to Analog Converter Board}

The digital to analog converter board has 4 separate DAC [9] channels and selected by an IC which is PPI 8255 and which is used to provide a stable analog set-Reference point input to the power supplies. This Reference input is given by the user with the help of GUI in LabView. This board is based on digital to analog converter AD767 (Analog Devices) [5]. The board layout is shown in Fig-3.

AD767 is a voltage output digital to analog converter which is 12-bit. It has on chip high stability buried Zener reference with +/- 1LSB (max) error. The input data is latched on rising edge of write pulse. The device is interfaced to 8bit micro-controller by using a PPI chip (8255).The analog outputs can be programmed using jumper configuration for 0 to $+5 \mathrm{~V}, 0$ to $+10 \mathrm{~V},+/-5 \mathrm{~V},+/-10 \mathrm{~V},+/-2.5 \mathrm{~V}$. All the ana$\log$ output channels are buffered by isolation amplifier AD210 (Analog Devices) to provide 3 port galvanic isolation.3-port isolation comprises isolation distances between the input circuit and the output circuit and also auxiliary energy. The auxiliary energy supply is neither located on the input potential or on the output potential; it is an independent galvanically-separated circuit. Due to the design of the 3 port isolation amplifier provides reliable protection against erroneous measurements owing to earth problems and parasitic voltages. 


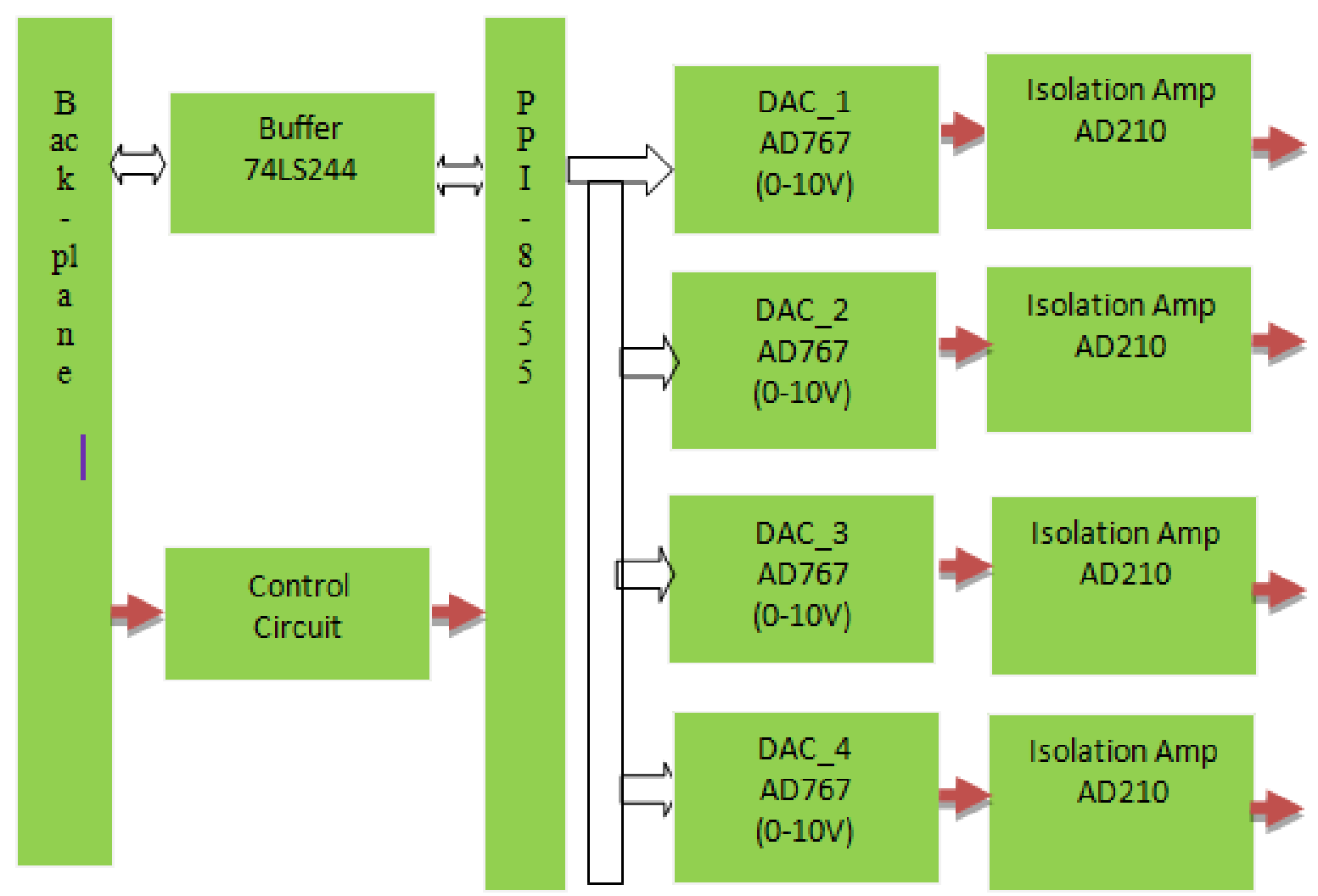

Fig 3: Digital to Analog Converter Board

The Port PA and upper portion of PB is configured as output port used to set DAC to obtain analog output, along with Lower portion of PC of 8255 used to enable one of the 4 DAC acts as chip select input to AD767 DAC IC.

\subsection{Analog to Digital Converter Board}

ADC board is used as an analog read-back output signal such that Power supply output is converted into digital signal with the help of this circuit to check whether reference to power supply is correctly set or not. The analog to digital converter board has 4 ADC channels. This board is based on analog to digital converter ICL71091 (Maxim). The block diagram of the board is shown in Fig-4.

The ICL7109 is a high performance, CMOS, low power integrating ADC. The ADC is dual slope integrating type. This ADC was selected because of its excellent linearity and noise rejection characteristics. In addition, the ICL7109; supports auto zero cycle for nullifying the effect of offset and its drift. The conversion rate of this ADC is 10 samples/sec and is satisfactory for this application as the signals are of very low frequency. The output data (12 bits, polarity and over-range) can be directly accessed through 8-bit $\mu \mathrm{C}$ bus. A PPI chip (8255) is used for interfacing ADC handshake signals (RUN/HOLD and SATUS) and channel select lines of the multiplexer. Here, decoder (74HCT138) is used to enable either 8255 when address of 8255 is passed, or to enable high byte and low byte of ADC. 


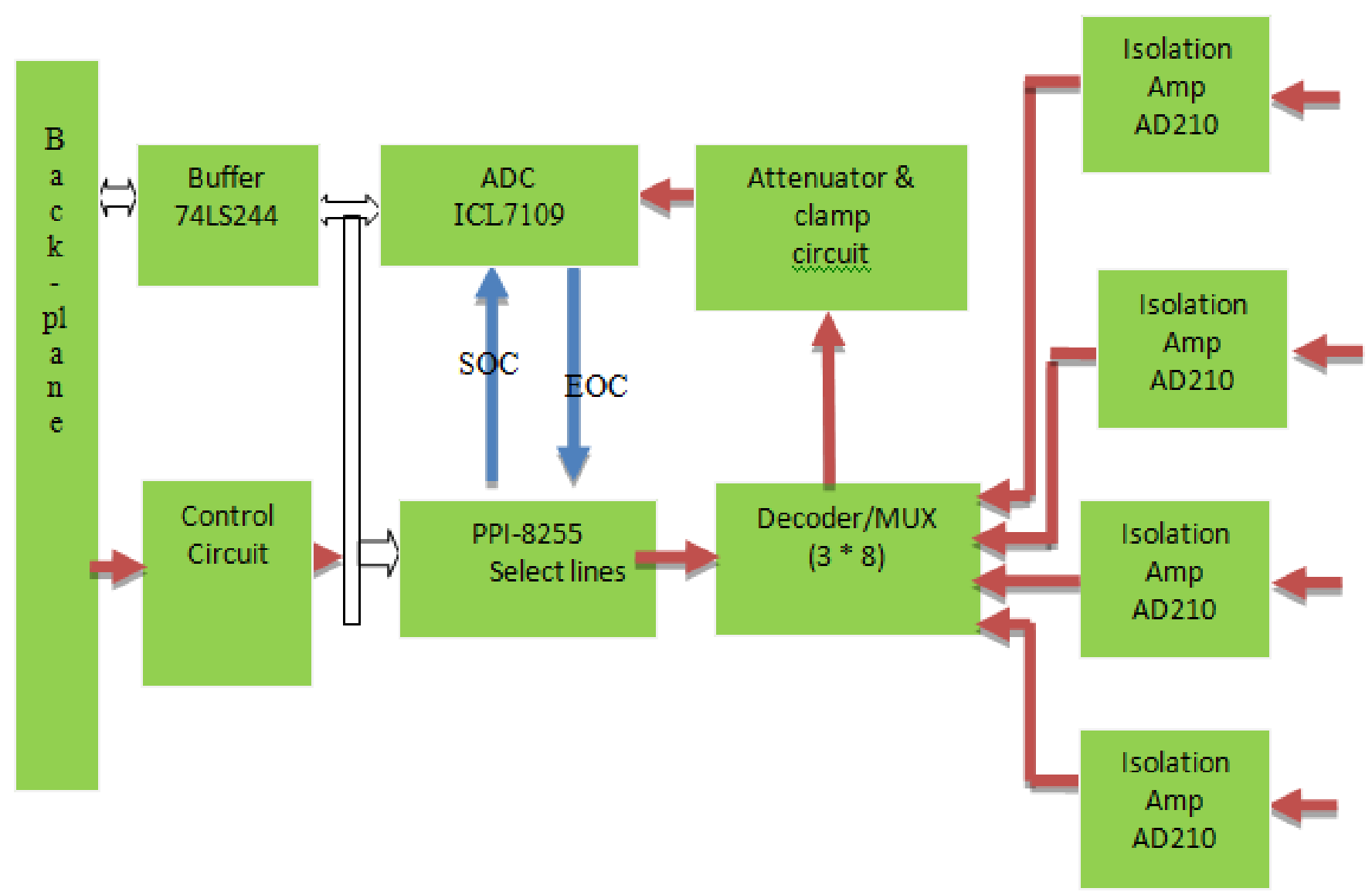

Fig 4: Analog to Digital Converter Board

PPI Port C lower pins PC0-2 consists of three lines (S0, S1 and $\mathrm{S} 2$ ) that help in channel select lines for the multiplexer. PC-3 is connected to the RUN/HOLD pin to issue start of conversion command to ADC. CPU monitors ADC busy status via PC-4 bit. Conversion can be initiated by pulsing RUN/HOLD signal high. The ADC indicates End of Conversion (EOC) by its STATUS output active low. Microcontroller monitors this status line continuously. When this line goes low, micro-controller reads 12-bit digital data. This data is converted to the appropriate measuring unit. This data is buffered for sending over to the RS-485 link to the master PC.

The analog voltages can vary over a wide range; hence the ADC card offers a wide dynamic range. The input voltage channels are protected by a protection circuitry. The card provides galvanic isolation for all the four channels.

\section{SOFTWARE SCHEME}

The basic software platform scheme for automation, Master/slave architecture is proposed. In the implementation, test software is developed in C [8] language using the KEIL cross compiler for Slave Module controllers. This program code is downloaded to the controller MCS-51 to communicate and access from remote located master PC through the RS-232/RS-485 serial port. And the front end Graphical user interface for master PC is developed in LABVIEW [7] which offers users to control and monitor the device parameters.

Firmware for slave controller is developed in ' $\mathrm{C}$ ' language [8]. In this work, various Modbus functions are written, which includes formation of query, slave identification, $\mathrm{read} / \mathrm{write}$ coil, read write registers, parsing of message, formation of response etc.

Power On sequence initializes various peripheral chips like serial port, ADC, DAC, PPI, LCD etc. All the digital as well as analog output signals are set to zero. The serial port was set to $19.2 \mathrm{Kbps}$ baud rate.

Functions are written for setting DAC, Reading ADC in interrupt mode, setting digital parameters, reading the digital status.The serial port receives message frame in interrupt so no request from PC will miss. Slave controller receives the frame up to the terminator, and then it calculates the LRC/CRC. It checks if the received LRC/CRC matches the calculated one, if yes then it displays the valid frame has received. Slave controller check the address received, if it is for the controller, controller parse the message for finding out the desired request. It takes appropriate action of request and the finally make the response for requested action and send the response to the PC. The main program flow is showed in Fig-5. 


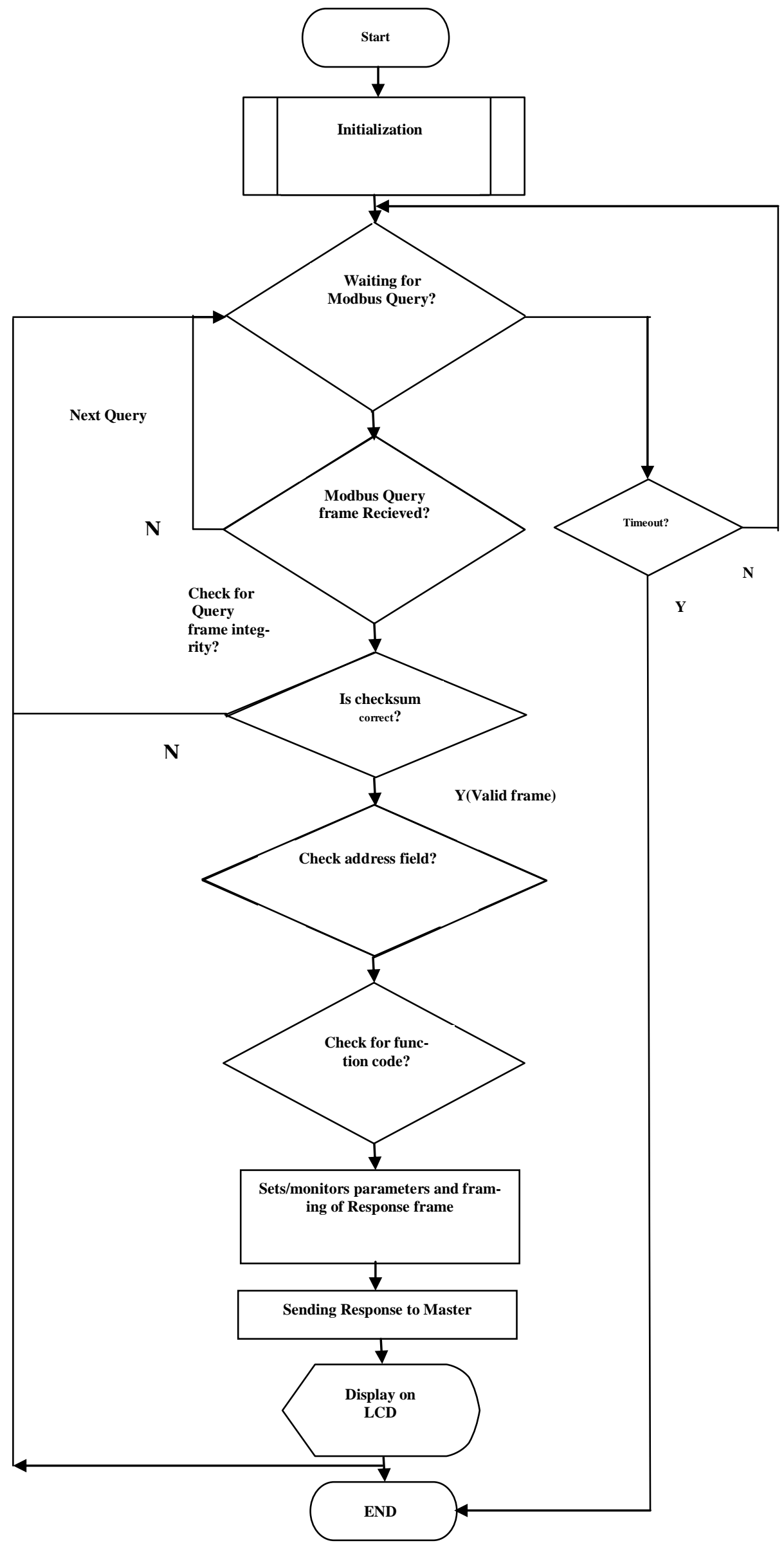

Fig-5: Slave controller firmware flowchart 


\section{SIMULATION SETUP}

For the smooth operation of remote operation various LabView panels were developed, which are described below.

\subsection{Main Panel (Welcome Screen):}

This panel provides the selection of slave controller to which user wants to communicate as shown in Fig-6.

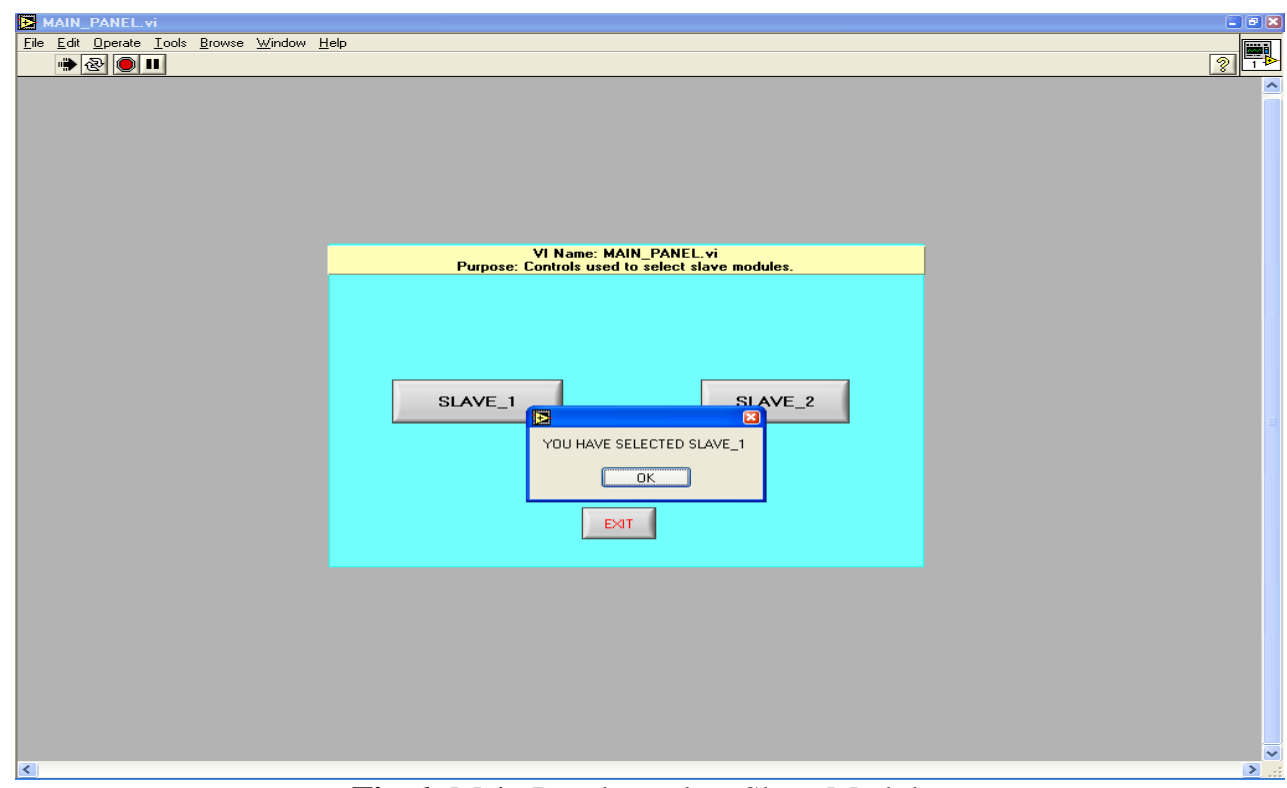

Fig-6: Main Panel to select Slave Modules

This window has two push buttons allows the user to select a slave within 2 slaves, as in this system only 2 slave are there and also having a EXIT button to close the GUI. When user selects particular slave, a message screen appears which wants acknowledgment for further proceed.

Now clicking on OK button the next sequence of frame structure executes and a new window launches to shows the detailed screen of selected slave. The entire main panel VI is developed under the frame structure of LabView.

\section{Front Panel for Slave Controller_1}

This VI is used to get the input query from the users. Likewise the front panel for other slaves is also same as shown for the slave_1.Front panel for slave controller 1 is shown in Fig-7.

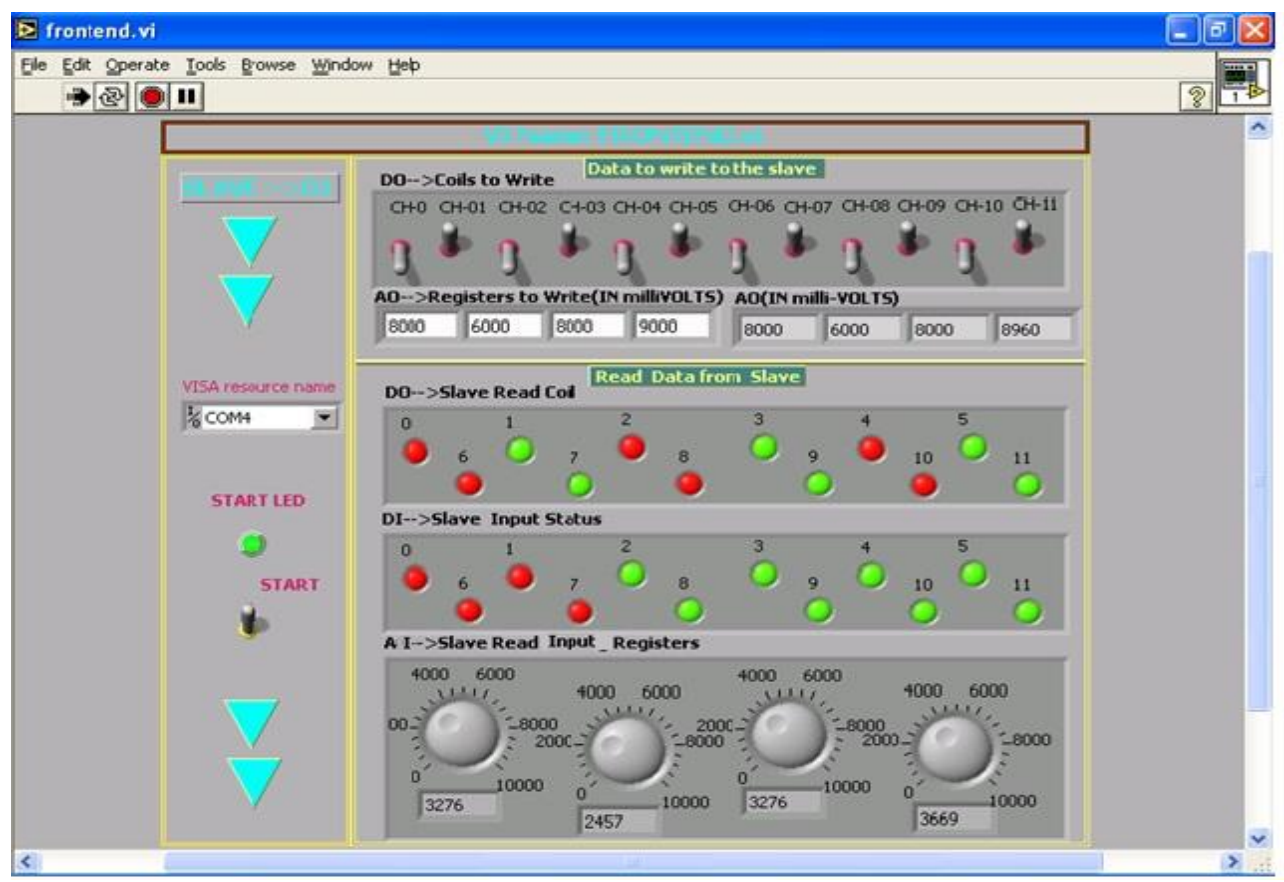


Fig-7: Front Panel for Salve_1

This panel has control buttons, which provides the user to set the query and also monitor the response on the same GUI.

On the front panel of GUI, the user first selects the serial port then set the query.

1. The user can make any power supplies ON/OFF/Reset by the Modbus function - Write coils(Digital Output $\mathrm{DO} \rightarrow$ Coils to Write on the GUI) through 12 toggle switches provided on GUI.

SLAVE 01:

Power supply 1: ON - CH0, OFF- CH1, RESET- CH2

Power supply 2: ON - CH3, OFF- CH4, RESET- CH5

Power supply 3: ON - CH6, OFF- CH7, RESET- CH8

Power supply 4: ON - CH9, OFF- CH10, RESET- CH11

2. The status of each of 12 digital outputs is monitored by the Modbus function- Read Coils (namely Digital Output DO $\rightarrow$ Slave Read coil on the GUI) and indicated by 12 LED's on GUI coming from the Digital Input Output board.

3. The Previous status of these power supplies i.e. ON/OFF/Reset are shown by 12 LED's provided on GUI which indicates the Modbus function-Read input status(namely Digital Input DI $\rightarrow$ Slave Input Status on the GUI)from the power supplies.

4. Also, the user can set reference voltage to each of the four DAC connected with each of four power supplies by the Modbus function- Write holding Registers (namely Analog Output $\mathrm{AO} \rightarrow$ Registers to write in $\mathrm{mV}$ on the GUI by using control buttons provided on GUI.

5. The status of these four DAC is monitored by the Modbus Function - Read Holding Registers (namely Analog Output $\mathrm{AO}$ in $\mathrm{mV}$ on the GUI) coming from the DAC board.

6. The Previous Read back power supplies voltage settings are shown using the Modbus functions-Read Input Registers (namely analog inputs $(\mathrm{AI}) \rightarrow$ Read Input Registers on the GUI) from the four Power supplies to the ADC board, indicated by analog dial indicators.

\section{CONCLUSION}

In this work a distributed Microcontroller based Control Scheme has been implemented for the remote control operation of the experimental setup of FEL and the user has successfully carried out the experiments.

The performance of the control system was found satisfactory which are as follows:

- The firmware on Slave controller is developed in 'C' language and PC software is developed in LabView 7.0 for making Graphical User Interface due to this implementation it enables the users to concentrate on the experiment and make effective use of the information displayed.

- The data logging features of the system were found highly useful to tune the setup for the repeatable operation and the diagnostic data was useful to pin point the operational faults in the early developmental stage.
- In this system, slave controllers which are interfaced to the master PC in a bus topology rather in star, with this implementation system overcomes with the associated drawbacks with the star configuration.

Although not formally standardized, it is also regarded as an open protocol as MODBUS ASCII/RTU. The physical layer of communication system is an RS-485 two-wire network, which provides the advantages like it is of low cost and sufficient data bandwidth according to application along with its simplicity as in the present case.

\section{ACKNOWLEDGMENTS}

The authors wish to thank Ms. Lalita Jain, SO ' $E$ ' in RRCAT, Indore for its support with all of the devices and equipment used in this project.

\section{REFERENCES}

[1]. Lalita Jain et. al. "Design and Performance of Supervisory Control System for RF Power Conditioning of Linac and Measurement of Transmission through Undulator", RRCAT Report, RRCAT/2008-2 http://www.cat.ernet

[2]. Karmens, D. M.; "Palms as HMIs in the Industry". VI Induscon.CDE-15, Joinville/SC, 2004

[3]. W. Kastner, G. Neugschwandtner, S. Soucek, and H. M. Newman, "Communication Systems for Building Automation and Control", Proceedings of IEEE, Vol. 93, Issue 6, June 2005, pp. 1178-1203

[4]. Modbus-IDA, (2006), "MODBUS Application Protocol Specification" Vl.lb [Online]. Available: http://www.modbus-ida.org/

[5]. Analog Devices Inc.., "Linear product data book", Analog Devices., 1988, p5-19. http://www.analog.com.

[6]. Referred the datasheets website from, http:// www.alldatasheet.com/

[7]. Labview Resources at National instruments, http://www. ni.com

[8]. "The 8051 Microcontroller and Embedded system using C" by Muhammad Ali Mazidi. -2nd edition

[9]. Referred the datasheets website from, http:// www.alldatasheet.com/ 